\title{
Determination of Unique feature set from Dental Image Graphs as Bio-Metric Information using Image Processing Techniques
}

\author{
Shrikanth Sharma ${ }^{1}$, Monika Aggarwal ${ }^{2}$ \\ ${ }^{1}$ (ECE Deptt. BGPC, Sangrur, India) ${ }^{2}$ (ECE Deptt., BGIET, Sangrur, India)
}

\begin{abstract}
A manual comparison relating to the AM and PM record is based on a deliberate dental chart completed by some forensic experts. With this chart, a number of distinctive features are renowned for each tooth individually. These functions include properties of the teeth (e.g., tooth present/not present, crown and root morphology and pathology and dental restorations), periodontal tissue features, and anatomical features. With regards to the number of matches, the forensic expert rejects or confirms the tentative identity. Unlike other biometric characteristics (e.g., fingerprints, iris, etc.), dental identification is complicated by the fact that dental features do change with time. Teeth can transform appearance, or might be missing altogether, due to dental work or accidents occurring as soon as the AM records are taken.

In the presented thesis work, emphasis is given on how authentically the dental image/radio graphs can be utilized in determining the identity of a person. The focus of the work is derived towards the finding of a set of unique features from dental radio-graphs and that is differentiable too from person to person. The shape (contour) of the teeth and their position in right or left jaws is one of the important information in the dental radio-graphs.
\end{abstract}

\section{INTRODUCTION}

The goal of forensic dentistry is to identify people based on their dental records, mainly as radiograph images. In the proposed thesis work, dental radio graphs are attempted to be treated as one of the bio-metric information of human being, with the final goal of human identification. The dental radiographs are more permanent rather than finger prints, iris or facial features of a person. Also, the dental radiographs remains available even after several hours of expiry of a person. This enforces the forensic science experts to use dental radiographs to be as a very important technique in fool proof identification of a person. A ranking of matching scores is generated based on the distance between the ante-mortem (AM) and post-mortem (PM) tooth shapes. Initial experimental results on a small database of radiographs indicate that matching dental images based on tooth shapes and their relative positions is a feasible method for human identification.

From a pattern recognition and computer vision standpoint, the problem of person identification based on dental record scan be cast as an image matching and retrieval problem: given a dental image. At a first glance, this problem seems more constrained than the generic object detection and object recognition problem. However, building a dental chart with distinctive features for each individual tooth (as human experts do) is extremely difficult, because the system must detect and classify each tooth before it can process them. One issue is the fact that the coarse shape of a tooth is not unique. For instance, all pre-molars have roughly the same shape, as do all molars and all incisors.

\section{BRIEF LITERATURE SURVEY}

Image segmentation is typically defined as the process of extracting objects from the image background. It is usually performed by subtracting the object location from the rest of the image [3]. This object location is usually calculated by an edge detection, an intensity measure or a target recognition algorithm [5].

However most of these techniques suffer from different types of noise due low resolution or poor lighting, which results in un-successful segmentation. Segmentation subdivides an image into it's constitute regions or objects. In the Dental Image perspective, segmentation is to recognize and label individual tooth in the X-Ray image or parts of the tooth such as crown and root of the tooth [4]. Each tooth or object extracted from the image represents Region of Interest (ROI) that contains important data used for later steps. ROI is defined as a rectangular part of the image that focuses on one object of the extracted objects from the image $[7,8]$. The following image represents an X-ray image and the specified object inside the rectangle represents the ROI $[1,3]$. In most of the segmentation algorithms, the segmentation is done either by extracting region based features, that can identify different objects and regions, or by applying a model and try to adjust its parameters to fit the processed objects or regions. Although the model based approaches are more complicated but they are more successful and reliable. 
The paper work is divided into following stages:

1. Dental Image Grabbing using X-Ray image Source. The focus of the proposed work is on determining the set of unique features from the dental images. Therefore, we propose to get the images for testing purpose directly from the dentists.

2. Noise Removal and Image Enhancement (Thresholding): The grabbed image is very noisy as being the $\mathrm{x}$ ray image, however gray in color. Some image processing techniques are applied to get a clear binary image.

3. Feature Identification: Contour, shape, counting of teeth, orientation, no. Of molar teeth and normal teeth, and their position in left, right, upper and lower jaws are some of the important features that may make a good set of features for identification. The proposed system overview is described in the below figure:

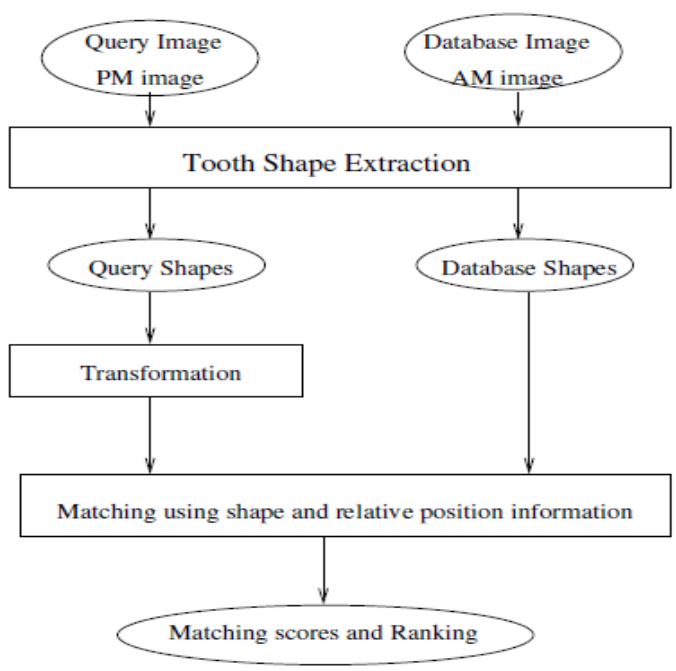

\section{IMAGE ACQUISITION}

The dental images are acquired by using the X-ray imaging system. In the presented work, the images are obtained from the local area dental clinic for reference purposes. The dental radiograph image (RGB image) so obtained is converted into a gray-scale image and a median filtering is performed to reduce noise. Below figures are the examples of the dental radio graphs.

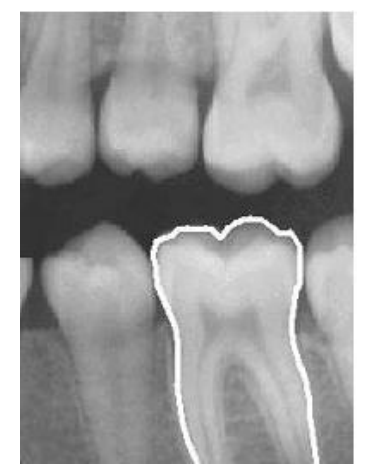

\section{IMAGE THRESHOLDING AND NOISE REMOVAL}

Histogram Equalization followed by Otsu algorithm is used for image thresholding. Once the image is binarized, the teeth are segmented based on pixel connectivity criteria. Say, for example on an average, the area of a tooth may be approximately100 sq. pixels unit. Then, the pixels groups of less than 100 may be left aside and may be discarded. However, the pixel groups of more than 100 sq. pixels unit may be treated as tooth and segmented. Once individual teeth are extracted. Dimensional features like area, contour, perimeter, max. and min. radii, standard deviation etc. are computed using statistical computation algorithm. 


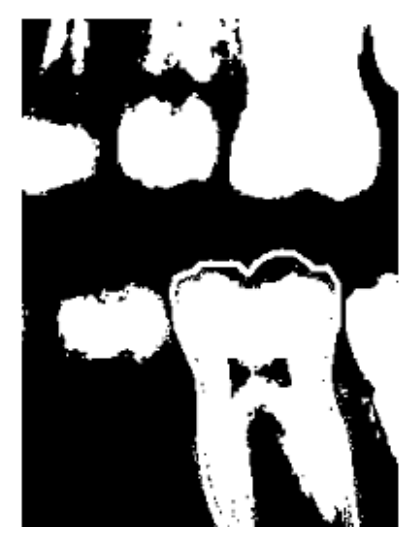

\section{IMAGE SEGMENTATION}

Dental radiographs are very firstly divide into four regions: Top Left, Top Right, Bottom Left and Bottom Right. For counting of teeth, the image is segmented using the bwlabel command in matlab. Following segmented images are obtained when bwlable command is applied on above shown figure.
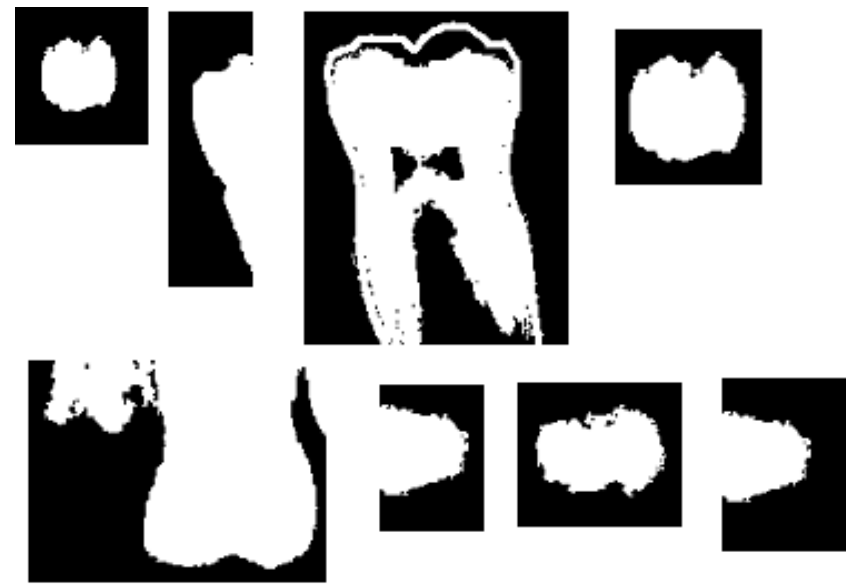

Segmented Images

The respective quadrant counts of teeth are obtained from the segmented images based on the location out of the four quadrants.

Once the images are segmented, it is easier to apply the pattern measurement algorithm.

The teeth dimensions i.e. perimeter, max. and min. radii in each quadrant, standard deviation, mean radius and, figure aspect are obtained by applying the statistical algorithm as discussed in next section.

\section{TEeTH PARAMETERS}

Following statistical features are computed from the teeth with respect to centre of gravity:

[a] Normalised Maximum Radii in each Quadrant represented by $R_{1}, R_{2}, R_{3}$, and $R_{4}$. See fig. (1)

[b] Intercepts on each axis represented by $X_{1}, X_{2}, Y_{1}$ and $Y_{2}$ with respect to centre of gravity of object. See below figure.

[c] Mean Radius $\left(\mathrm{R}_{\mathrm{M}}\right)$

[d] Figure Aspect i.e. length to width ratio (FA)

$$
\mathrm{FA}=\left(\mathrm{X}_{1}+\mathrm{X}_{2}\right) /\left(\mathrm{Y}_{1}+\mathrm{Y}_{2}\right)
$$

[e] Normalised Perimeter $(\mathrm{Np})$

$N_{p}=$ Total no. of pixels at the contour of object / $R_{M}$

[f] Normalised Standard deviation of radii taken from centre of gravity of object (NSD).

$\mathrm{SD}=\sqrt{ }\left[\left(\mathrm{Ri}-\mathrm{R}_{\mathrm{M}}\right) 2 / \mathrm{N}_{\mathrm{p}}\right]$

$\mathrm{NSD}=\mathrm{SD} / \mathrm{R}_{\mathrm{M}}$ 
Where $R_{M}$, and $R_{i}$ are the mean radius and $i_{\text {th }}$ radius i.e. distance of $i_{\text {th }}$ pixel on contour of the pattern from its centre of gravity.

[g] Normalised area (NA) of the pattern.

Normalised area $=$ Total pixels on objects $/ \mathrm{R}_{\mathrm{M}}{ }^{2}$

All features are normalized with respect to mean radius of the pattern ${ }^{[6]}$. It makes all the statistical features independent of size of the pattern. The set of described statistical features may be termed as figures of merit to classify an object.

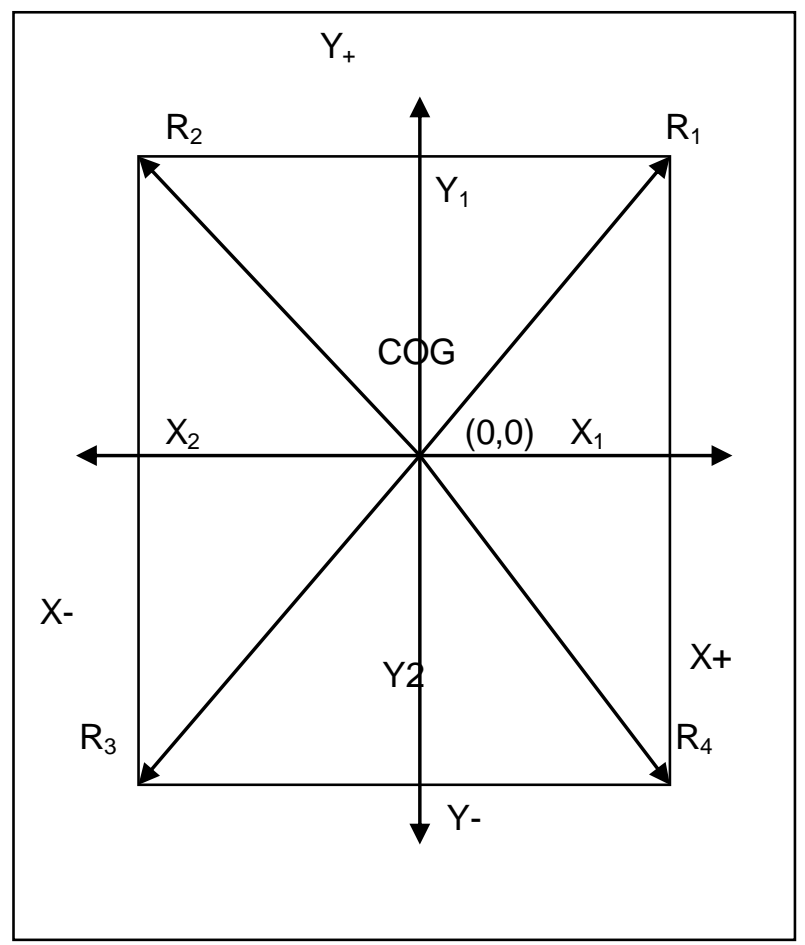

Following parameters are extracted for any teeth segmented from the image:

1. Min. Radii in each quadrant

2. Max. Radii in each quadrant

3. Mean radius

4. Perimeter

5. $\quad$ Figure Aspect

6. Standard Deviation

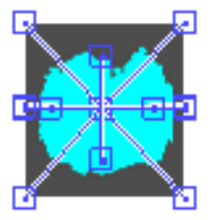

Besides above, the no. of teeth in each quadrant of the jaw is also obtained along with its respective location in the jaw.

Following parameters may be attributed to a jaw of a person in affixing his dental biometrics identity:

1. No. of teeth in top left jaw

2. No. of teeth in top right jaw

3. No. of teeth in bottom left jaw

4. No. of teeth in bottom right jaw

5. Max. perimeter teeth and it respective location in the respective quadrant

6. Min. perimeter teeth and it respective location in the respective quadrant 
7. Max. Area teeth and it respective location in the respective quadrant

8. Min. Area teeth and it respective location in the respective quadrant

9. No. of missing teeth in each quadrant along with its respective location

10. Max. Perimeter teeth and its location

11. Min. Perimeter teeth and its location

12. Max. Area teeth and its location

13. Min. Area and its location

14.

\section{CONClusion}

In Forensic Dentistry, human experts generally perform manual comparisons of ante-mortem and postmortem dental records, looking for differences among teeth patterns. Despite its accuracy, this manual comparison method demands great amount of time, which is not admissible in large scale identification. Therefore, the development of techniques and systems that facilitate human identification through automated teeth recognition is required.. The results obtained with the statistical parameters and no. of counts can be considered good, when the high variability of this biometric characteristic is observed. As the statistical parameters are considered in identification of the teeth, the statistical parameters do not vary to a high degree to slight variation in the teeth shape occurred due to imaging device system. Therefore, a fair degree of accuracy may be obtained using the discussed parameters in affixing the dental biometrics identity of a person.

\section{ACKNOWLEDGEMENTS}

We are thankful to Mr. Vikas Goel, Sr. Project Manager, C-DAC, Mohali, Punjab for his valuable guidance and continuous support in making this paper.

\section{REFERENCES}

[1] Robert Howell, “Dental Remains”, Dental Task Force Technical Report by the Criminal Justice Information Service, Federal Bureau of Investigation on Forensic Identification.

[2] Integrated Automatic Fingerprint Identification System (IAFIS), Technical report by the Federal Bureau of Investigation, Criminal Justice Information Services Division, March 5th 2002.

[3] P. Stimson \& C. Mertz, Forensic Dentistry. CRC Press 1997.

[4] M. J. Smith, A. Docef, “ A Study Guide for Digital Image Processing”, SP. Inc., 1999.

[5] J. C. Goswami and A. K. Chan: Fundamentals of Wavelets, Theory, Algorithms and Applications. A Wiley-Interscience Publication, John Wiley \& sons, Inc. 1999.

[6] Y. Zhang, M. Brady, S. Smith, "Segmentation of Brain MR Images through a Hidden Markov Random Field Model and the Expectation Maximization Algorithm, IEEE Trans. Medical Imaging, Vol. 20 No.1, 2001.

[7] V. Metzler, C. Thies, T. Lehmann and T. Aach, "Segmentation of medical images by morphological scale-space filtering", Medical Imaging 2001, Procs. SPIE 4322, pp. 139-150, 2001.

[8] D. J. Michael and A. C. Nelson, "HANDX: a model-based system for automatic segmentation of bones from digital hand radiographs" Whitaker Coll. of Health Sci. \& Technol., MIT, Cambridge, $\quad$ MA; To appears in: Medical Imaging, IEEE Transactions 